The coexistence of malignant tumour and pregnancy is a state of simultaneous occurrence of two completely contradictory philosophical and biological phenomena - the development of a new life and a life-threatening terminal illness. Finally, a physician - in fact the whole team of doctors - is facing the fight for two lives: of the mother and her unborn child.

The incidence of malignant disease in pregnancy is 0.05 to $0.1 \%$. This condition is a major challenge for physicians because there are no randomised studies that could be the basis to choose the therapeutic methods - the medical knowledge merely comes from case reports, registries, and observational studies.

The following cancers most often coexist with pregnancy: gynaecological neoplasm (especially cervical and ovarian cancer), breast cancer, lymphatic system neoplasm, and melanoma.

Formerly, the diagnosis was clearly the necessity of abortion. Today - although unskilled doctors still propose the only therapeutic option - termination of pregnancy is not the only solution.

The past few years have seen the updating of reports and guidelines for the management of pregnant women with cancer. This paper is a review and summary of the information from these publications.

Key words: cancer during pregnancy, chemotherapy, cervical cancer, ovarian cancer, endometrial cancer.

Contemp Oncol (Pozn) 2016; 20 (3): 193-198 DOI: $10.5114 /$ wo.2016.61559

\section{Gynaecological cancers coexisting with pregnancy - a literature review}

\author{
Anna Skrzypczyk-Ostaszewicz ${ }^{1}$, Maryna Rubach ${ }^{2}$
}

${ }^{1}$ Department of Oncological Diagnostics and Cardiooncology, Oncology Centre The Maria Skłodowska-Curie Institute, Warsaw, Poland

${ }^{2}$ Department of Oncology and Internal Medicine, Chemotherapy Day Ward, Oncology Centre - The Maria Skłodowska-Curie Institute, Warsaw, Poland

\section{Introduction}

The incidence of malignant tumours, particularly epithelial, increases with age, and consequently most cases are observed between 50 and 60 years of age. However, some solid tumours are likely to appear in the reproductive age, and thus it is possible that they will occur during pregnancy [1].

The coexistence of cancer and pregnancy can delay making a diagnosis, especially for those with breast cancer. It is more difficult to assess the effect of pregnancy on prognosis in malignant tumours - the available data on the subject are very limited and sometimes contradictory [1].

Malignant tumours in pregnant women are very rare, but they are a serious problem - women are increasingly postponing the decision about motherhood (the age of primapara in Europe increased in the last decade by two years on average), so we should expect a growing number of such cases during pregnancy [2].

The treatment of malignancies in pregnant women is difficult. Diagnostic and therapeutic procedures should be carried out carefully, taking into account the risk factors associated with both woman and foetus. The optimal management should include the proper assessment of oncological and obstetric risk, clear decision-making process - both for the patient and for the child, the appropriate treatment, and careful observation after its completion, based on close interdisciplinary collaboration between an oncologist, obstetrician, and neonatologist [2].

\section{Gynaecological cancers coexisting with pregnancy}

The treatment of gynaecologic cancers complicating pregnancy entails the need to consider the maintenance of pregnancy but also the potential loss of the ability to reproduce as a result of the conducted therapy.

The most common gynaecological neoplasms diagnosed during pregnancy are cervical and ovarian cancer. Endometrial cancer or cancer of the vulva very rarely coexist with pregnancy [3].

\section{Cervical cancer during pregnancy}

The incidence of cervical cancer in pregnant women is not explicitly evaluated - the retrospective data took into account both invasive and pre-invasive cancers, and in other cases also cancers diagnosed after birth. Undoubtedly, it is the most common cancer in pregnancy among all gynaecological malignant growths [3-5].

It is accepted that cervical cancer occurs in 1-12 cases per 10,000 pregnancies. Pregnancy does not have a negative impact on prognosis and the therapeutic outcomes are the same as in non-pregnant women $[3,5]$. 
Screening for cervical cancer is an important part of prenatal care. Many guidelines recommend performing the Papanicolaou (Pap) test in all women at the moment of diagnosing pregnancy, although its interpretation during pregnancy can be difficult due to frequent erosions, inflammations, and the presence of decidual cells, which might be confused with atypia (the so-called Arias-Stella reaction). Generally, the test has the same importance as in patients who are not pregnant [3].

After finding the abnormal result of the Pap test, diagnostic procedures should be the same as in non-pregnant women. Colposcopy with biopsy is characterised by a sensitivity of $73-95 \%$. Curettage of the uterine cervix is contraindicated during pregnancy [3].

The changes of low degree regress in about $60 \%$ cases and remain unchanged in about $30 \%$. The progression to more advanced changes (CIN3) is rare and occurs in a maximum of $6 \%$ of patients. In disorders of high degree, such as CIN3, the percentage of regression is low - about $30 \%$, and progression involves approximately $10 \%$ of patients. If there is no evidence of invasion, no treatment should be implemented during pregnancy and the entire therapy can be postponed until after the birth. In patients with no invasive changes, monitoring is recommended using colposcopy performed in each trimester with repeated biopsy in cases suspected of progression $[3,5]$.

The indications for conisation in pregnancy are more limited, due to the risk of severe complications of surgery such as bleeding (including death) in up to $15 \%$ of patients, miscarriage, premature labour, or premature rupture of membranes. This risk increases with the advancement of gestation, thus if there is a suspicion of micro-invasive or invasive changes, conisation or excision of the changes using a large diathermy loop should be carried out in early pregnancy. The choice of therapeutic method depends on the dimensions of the cervix, the preferences of the gynaecologist, and the expected progression of pathology. The choice of treatment must always be discussed with the patient and should be consistent with the patient's beliefs [3].

The management in the case of invasive cervical cancer depends on: 1) the size of tumour, 2) the state of the lymph nodes, 3) the stage of pregnancy, and 4) the histological subtype [3].

The basic histological subtypes, i.e. squamous cell carcinoma, adenocarcinoma, and glandular-squamous carcinoma, have similar prognosis and should be similarly treated, as opposed to the more rare and poor prognosis type, i.e. small cell carcinoma. In the latter case it is necessary - if possible - to terminate pregnancy and the patient should be immediately subjected to the optimal therapy [3].

In the past, abortion and radical therapy of cancer was the treatment of choice in the case of cervical cancer in the first and second trimester of pregnancy. At present, the aim is to maintain pregnancy, particularly in patients with the disease at an early stage, without lymph node involvement. Staging, including the status of regional lymph nodes, must be properly carried out. Magnetic resonance imaging (MRI) is the best imaging technique for this pur- pose, which can be performed without contrast in the evaluation of cervical cancer [3-6].

Balleyguier et al. reported 12 cases of pregnant women assessed using MRI. In all the cases, the examination was conducted before conisation using the camera 1.5-T. The patients were examined in the supine position and did not receive any contrast agent. Two radiologists evaluated the following: the local condition (parametritis, uterus, bladder, and rectum), the visibility of changes, the size of changes, and the intensity of signal compared to the uterine and cervical muscles as well as the lymph nodes of the abdomen and pelvis. In addition, the test took into account imagining problems caused by the movements of the foetus. After determining the degree of progression, making therapeutic decisions, and implementing the treatment, the control MRI examinations were repeated every four weeks until the $30^{\text {th }}$ week of gestation $[3,7]$.

The guidelines of the European Symposium on Urogenital Radiology state that it is possible to provide a contrast agent (gadolinium derivative) in a pregnant woman, which can improve the visualisation of small lesions in MRI. The most up-to-date guidelines describe the gadolinium as possible to use only if it is absolutely essential $[6,7]$.

The features of cervical cancer in MRI during pregnancy are similar to those found in patients who are not pregnant. However, we ought to remember that in non-pregnant women, cervical tumours are usually hyperintense compared to the muscles of the uterus, while in pregnancy the normal cervix can be hyperintense, causing the tumour to look isointense or even hypointense. In all of the described cases, MRI had a significant impact on the therapeutic decisions $[3,7]$.

The PET-CT examination, which is used to assess the extent of lesions in locally advanced cervical carcinoma, is contraindicated in pregnancy. This is firstly due to the exposure to a high dose of ionising radiation (CT) and because of the higher uptake of the radionuclide in the liver of the foetus with a longer retention than in the maternal liver (PET). This was demonstrated in animal studies (pregnant monkeys) $[3,5,8]$.

Histopathological evaluation of the lymph nodes remains the gold standard in the assessment of regional lymph nodes. The involvement of lymph nodes affects therapeutic decisions and can change the course of pregnancy $[3,4]$.

Laparoscopic lymphadenectomy is possible between the $13^{\text {th }}$ and $22^{\text {nd }}$ weeks of pregnancy [6]. The Society of American Gastrointestinal and Endoscopic Surgeons (SAGES) has developed guidelines describing laparoscopic procedures in pregnant women. Pelvic lymphadenectomy is a valid diagnostic procedure in the first and second trimester of pregnancy in patients with early cervical cancer. The rate of involved pelvic lymph nodes is comparable to that seen in non-pregnant women at the same level of progression. The histological evaluation of the lymph nodes collected from pregnant patients should be carried out by a qualified and experienced pathologist because some of the changes in the pelvic lymph nodes associated with the development of decidua can mimic metastasis to the lymph nodes, especially in squamous cell carcinomas. 
The immunohistochemistry test (with the assessment of cytokeratins) allows the disease to be distinguished from physiological changes occurring during pregnancy [3].

Sentinel node detection using radiocolloid is not recommended in pregnant women with cervical cancer, although some researchers believe that this procedure is technically feasible in this group of patients [3, 4, 9].

Approximately $70 \%$ of cervical cancers coexisting with pregnancy are detected at stage I according to FIGO. At this stage, if there are no metastases to the lymph nodes, there are two possible methods of management: 1) close clinical observation (in order to rule out tumour progression) and the postponement of treatment until maturity of the foetus; then the treatment is implemented after birth, 2) sparing surgical treatment of cervical tumour to preserve the uterus and pregnancy [3].

At stage IA, the first method of treatment is associated with a very good prognosis. In this stage conisation alone is also sufficient and is a relatively safe procedure. The laparoscopic evaluation of the lymph node status is probably the best criterion for proceeding at stages IB1 - in the case of negative lymph nodes, patients with tumours smaller than $2 \mathrm{~cm}$ may be subjected to careful observation and implementation of treatment after birth; either simple trachelectomy or large conisation should be considered [3-5].

In locally advanced cervical cancer, a decision is taken about neoadjuvant chemotherapy or chemoradiotherapy. In pregnant women, the choice of this therapeutic method entails the need to terminate pregnancy prior to the implementation of therapy. In exceptional cases, where surgical abortion is not feasible (e.g. due to the large mass of cervical tumour), radiation therapy can be started during pregnancy. This results in spontaneous abortion within three weeks [3].

Neoadjuvant chemotherapy (NACT) alone and the maintenance of pregnancy is an alternative to the combined treatment; in such cases, surgical treatment (or chemoradiation) is implemented after birth. In stage IB2 and higher NACT is the only possibility to preserve the pregnancy. Most women described to date have been treated with cisplatin and paclitaxel $\left(175 \mathrm{mg} / \mathrm{m}^{2}\right)$. Cisplatin may be administered every seven days at a dose of $20-50 \mathrm{mg} / \mathrm{m}^{2}$ or every 21 days at a dose of $50-100 \mathrm{mg} / \mathrm{m}^{2}$ (usually $75 \mathrm{mg} / \mathrm{m}^{2}$ ) the second scheme is currently considered the standard for the treatment in pregnancy. There are also several reports on alternative using carboplatin (AUC 5-7.5), which may be an option to reduce nephrotoxicity, especially in patients with a positive history of pre-eclampsia $[3,5,6,9]$.

The patient should be presented with all therapeutic options, be aware of the risks, and take an active part in the final decision-making.

\section{Ovarian cancer during pregnancy}

Most of the changes detected in imaging studies during pregnancy in the uterus or ovaries are benign and are usually detected at a routine ultrasound check-up during the pregnancy observation [3, 5, 6, 10,11].

Sometimes these changes manifest themselves suddenly, giving symptoms of acute abdomen in the course of ovarian torsion, rupture or intraperitoneal bleeding $[3,5]$.
Diagnosis and treatment of a pathological mass found in the appendages depend on the radiological image. UItrasound is the most common examination. Simple ovarian cyst does not require any intervention - it usually absorbs spontaneously [3, 6, 10, 11].

There are different scales and ultrasound evaluation systems of pathological lesions, but it is not certain whether their value is the same as in pregnancy practice. A suspicion of malignant nature of the change is based upon: the size (the changes measuring $5 \mathrm{~cm}$ and more require surgical intervention), morphological assessment of the tumour, and the presence of changes outside the ovary. It may be useful to use the Colour-Doppler imaging technique in order to obtain a picture of the blood vessels and thus improve the quality of ultrasound $[3-6,10]$.

Computed tomography of the pelvis is contraindicated because of the high levels of ionising radiation and the associated risk to the foetus. Magnetic resonance imaging is preferred to accurately assess the pelvis during pregnancy $[3,4,6,8,10]$.

In the case of ovarian cancer, assessment of the marker CA-125 level is important in making a diagnosis. However, one must remember that during pregnancy it may be (like other tumour markers) physiologically elevated, especially in the first trimester (with a dozen or so per cent of pregnant women the level of CA-125 may be higher than $65 \mathrm{IU} / \mathrm{ml}$ ). The level of CA-125 normalises during the second and third trimester, but it grows rapidly immediately after birth. The return to the starting level occurs within 2-10 weeks postpartum. Therefore, the evaluation of CA-125 marker in pregnant women is not helpful in the first trimester of pregnancy, but it can be useful in the second and third trimester [3, 4, 8].

Elevated levels of hCG, AFP, and SCC also occur during normal pregnancy. Some authors suggest that inhibin B, $A M H, H E 4$, and LDH physiologically should not be elevated and can be used in oncologic assessment [4]. But we should also remember that higher levels of tumour markers are associated with non-oncological obstetric complications such as miscarriage, preeclampsia, or HELLP [10].

In 1 out of 600-1500 pregnancies, a pathological change in the appendages is reported, which requires surgical intervention. Approximately $3 \%$ of these patients are diagnosed with malignant tumour of the ovary; however, non-epithelial tumours have been most commonly observed (embryonic and of the sex cords origin) - tumours of low-grade. Epithelial cancers follow them in incidence [3, 8, 11].

The treatment depends on the size of tumour, the morphological image (assessed using imaging testing or during surgery), histological subtype, progression of the disease, the course of pregnancy, and the expectations of the patient. The use of laparoscopic techniques can reduce the perioperative mortality rate, but we should remember that these procedures should be performed in compliance with all the standards designed to prevent the spread of tumour cells during surgery. The ideal procedure is to collect the specimens from the peritoneum together with the removal of the ovary and Fallopian tube on the affected side with the analysis of the frozen fragments - further investigation depends on the outcome [3-5]. 
Ovarian tumours of low malignant potential have a very good prognosis and most patients are treated only surgically, without chemotherapy. The vast majority of these tumours are diagnosed at stage I according to FIGO. The treatment of these tumours is the same as in non-pregnant women. If the surgical exploratory evaluation finds serous ovarian tumour of low malignant potential, it is necessary to resect the tumour and all grossly visible abnormal changes. If the contralateral ovary is macroscopically normal, it should be left [3-5].

In the absence of macroscopic peritoneal involvement, the routine assessment of progression should be carried out without lymphadenectomy (cytology, random peritoneal specimens, resection or biopsy of the omentum, appendectomy in the case of mucous tumours). All changes found outside the ovary should be removed in order to assess the progression and histological subtype [3].

Another surgical evaluation should be carried out in the case of serous tumours of low malignant potential with the accompanying micropapillary tissue. This subtype is characterised by the frequent occurrence of invasive implants outside the ovary. This procedure may be deferred for a period of time after birth provided that the disease is diagnosed after the $20-24^{\text {th }}$ week of gestation. Before the $20^{\text {th }}$ week of pregnancy, the laparoscopic evaluation seems to be the safest option [3].

In the case of malignant ovarian tumours, most patients have indications for chemotherapy. The maintenance of pregnancy and chemotherapy should always be individually discussed - the final decision depends on the biological features and the stage of the disease (histological subtype, differentiation, progression, including the involvement of the lymph nodes, tumour size, the presence of the disease in the peritoneum) and of course, as always, the will of the patient $[3,4,10]$.

In most patients with non-epithelial cancer (embryonal or of the sex cords origin), the tumour is of considerable size, which may exceed $30 \mathrm{~cm}$. These patients report more complaints than patients with epithelial tumours, including pain, bloating, and symptoms of acute abdomen. Over $90 \%$ of patients are diagnosed at the first stage. In order to take a decision to perform the surgery maintaining fertility, a full evaluation of the progression inside the peritoneal cavity should be carried out. Lymphadenectomy is not indicated, although the suspicious lymph nodes should be removed. Indications for adjuvant chemotherapy and the treatment regimens are similar to those in non-pregnant women - BEP (bleomycin, etoposide, cisplatin) is the program of choice $[3,5,10]$.

Epithelial ovarian cancers are rare in pregnant women and their treatment poses the most difficulties. The prognosis in these tumours is worse than in non-epithelial, in two thirds of patients there is the spread to the peritoneum and the lymph nodes and most patients require chemotherapy. Standard adjuvant chemotherapy should be considered for high-grade stage I and any stage II disease $[3,5,6,9]$.

The surgical treatment is complicated because it should include the resection of appendages, peritoneal metastasis, and lymph nodes. If a decision is taken to maintain pregnancy, the preservation of the uterus with evaluation of the disease progression in the peritoneal cavity is a primary procedure that should be offered. During the initial surgical procedure, only the suspicious lymph nodes should be removed. The invasive cytoreductive surgical treatment leads to the termination of pregnancy because of the need for hysterectomy - such a procedure should be proposed when the diagnosis of epithelial ovarian cancer is made at stage III during the first or second trimester of pregnancy $[3,9]$.

In some patients with peritoneal dissemination, it is possible to maintain pregnancy and use neoadjuvant chemotherapy (carboplatin and paclitaxel) - there was no damage to the foetus if chemotherapy was administered in the second and third trimester. However, the observation of patients after the treatment described in the available reports is very short $[3,4,6,8,9,10]$.

The risk of birth defects as a result of chemotherapy administered during pregnancy is high in the first trimester of pregnancy, especially between the $2^{\text {nd }}$ and $8^{\text {th }}$ weeks of pregnancy, and therefore therapeutic abortion and the need to defer the treatment should be discussed with each patient. The cytostatic treatment of ovarian tumours in the second and third trimester of pregnancy should be in accordance with the standards applicable in non-pregnant patients, both in the case of non-epithelial and epithelial tumours. Usually, the premature termination of pregnancy is not necessary if the disease is well controlled with chemotherapy. However, delivery should take place in the period without the use of chemotherapy. Previously published reports described only single cases of birth defects and their relationship with chemotherapy is not clear $[3,11]$.

Most reported cases concern the safe use of bleomycin, carboplatin, taxanes, and vinca alkaloids. Newborns demonstrated neutropaenia, hair loss, and impaired hearing due to the use of cisplatin within three weeks before childbirth. Etoposide given in the third trimester of pregnancy caused transient neonatal pancytopaenia. A case of ventriculomegaly was reported in a newborn of a mother treated with BEP regimen. There are no reports on the use of topotecan in pregnant women, and data concerning gemcitabine are very scarce - it is suspected of having the ability to inhibit intrauterine growth $[3,11]$.

Targeted therapies are contraindicated in pregnancy. There are no reports on the intravenous use of bevacizumab in pregnant women. There are only a few reports of the application of this antibody during pregnancy in ophthalmic treatment without a negative impact on the course of the pregnancy and the development of the foetus. One paper describes the loss of early pregnancy after intraocular injection of bevacizumab. Certainly, however, the systemic administration of anti-angiogenic therapy cannot be recommended during pregnancy $[3,4,8]$.

\section{Endometrial cancer}

The coexistence of pregnancy with endometrial cancer is very rare. The data on this topic come from individual case reports, of which at least half were diagnosed in the 
course of uterine bleeding after childbirth. In other cases, the diagnosis was made while determining the cause of miscarriage. In most cases, well-differentiated cancer was observed without or with minimal invasion of the myometrium $[5,8,11,12]$.

Endometrial cancer diagnosed before fertilisation changes the intrauterine environment to one that makes fertilisation extremely difficult; on the other hand, pregnancy has a protective effect on endometrial tumour growth. It is suspected that the rare phenomenon of the coexistence of pregnancy and endometrial cancer may correspond to the presence of immature endometrium, resistant to progesterone. Cancer can occur focally in the immature basal cells, which do not respond to hormonal stimulation [8].

The radical removal of reproductive organs is the standard treatment of endometrial cancer, but in the majority of patients with endometrial cancer at stage I according to FIGO, the long-term prognosis is successful, and after discussing with them further maternity plans, they can undergo sparing therapy (curettage with the subsequent regular check-up with endometrial biopsy) [5].

\section{Vulvar cancer}

This malignant tumour is very rare in premenopausal women, although the incidence of invasive squamous cell carcinoma of the vulva in women under 40 years of age is growing due to HPV and HIV infections [4, 5, 8].

Radical vulvectomy with inguinal lymphadenectomy is the basic therapeutic option in cancer of the vulva in pregnancy. Sentinel lymph node biopsy during pregnancy should be used very carefully. Data about the safety of this procedure are very limited. The use of blue dye is not recommended during pregnancy due to a high risk of severe allergic reactions. Undergoing surgery is a relative contraindication to vaginal birth $[4,5]$.

Less extensive treatments are laser therapy or surgical excision of the changes like vulvar intraepithelial neoplasia (VIN) or partial vulvectomy with the sentinel node procedure in the case of invasive cancers with clinically non-suspicious regional lymph nodes [5, 9].

The treatment of vulvar cancer in pregnant women is not different in principle from that performed in non-pregnant women, but the advancement of pregnancy is important and should be taken into account before taking the final medical decision. In the third trimester, vascularisation of the vulva significantly increases, which also increases the operational risk (high mortality rate); therefore, if a diagnosis is made after the $36^{\text {th }}$ week of pregnancy, surgery should be postponed until after the birth [6].

If the inguinal lymph nodes are involved, the prognosis is unfavourable and immediate treatment is crucial. Here, the benefits of chemotherapy are small [9].

Vulva melanoma in pregnant women should be treated in the same way as in non-pregnant. Patients with poor prognosis should be informed of the high risk of recurrence, the possibility of death and the high risk of metastasis appearance in the foetus (22\%) [9].

\section{Vaginal cancer}

Primary carcinoma of the vagina is a rare gynaecological cancer, more often observed as secondary to the involvement of the vulva, cervix, endometrium, bladder, colon, or rectum. Histologically, primary vaginal cancers are squamous cell (up to 90\%), adenocarcinomas (up to 10\%), and occasionally melanomas and sarcomas - leiomyosarcoma and rhabdomyosarcoma [8].

It is believed that intrauterine exposure to diethylstilbestrol can cause clear cell carcinoma of the vagina in young women [8].

The coexistence of vaginal cancer and pregnancy is very rare and described in casuistic reports [8].

Vaginal tumours can cause discharge and irregular bleeding (usually contact bleeding). The microscopic evaluation includes the assessment of smears and biopsies taken during colposcopy. In order to assess the severity of the disease in pregnant women, the following examinations can be safely performed: cystoscopy, procto-rectoscopy, abdominal ultrasound, and conventional chest $X$-ray with abdominal protection. Because of the rarity of the disease, there are no guidelines for dealing with pregnant women. The treatment should be planned individually, depending on the progression of cancer, the stage of pregnancy, and the possibility of its maintenance. Childbirth, if it occurs, should be carried out, of course, by caesarean section [8].

It is not known whether the postponement of treatment due to pregnancy significantly changes the prognosis in patients with this diagnosis - regardless of the coexistence of pregnancy, advanced disease has a decidedly poor prognosis and the five-year survival rate is low [8].

\section{Summary}

The diagnosis of malignant tumour in pregnancy is a dramatic event that overshadows the joy of the forthcoming motherhood. Usually, these women are diagnosed with genital cancer, breast cancer, lymphatic system neoplasm, or melanoma. Other solid tumours are much less common during pregnancy [12].

Historically, the diagnosis was associated with the need to terminate the pregnancy. Today, although unskilled doctors still propose the only therapeutic option, abortion is not the only solution.

Thanks to the efforts of many experts, reports in recent years have been summarised and collected creating guidelines that would enable, at least in some cases, the maintenance of pregnancy and the effective treatment of cancer, especially gynaecological cancers and breast cancer.

Of course, the final decision is made by the patient. The patient should have the opportunity to decide about the fate of her pregnancy and it should not be affected by the moral beliefs of the doctor. The task of the professionals, who look after such a special patient, is to provide the woman with access to the most current and reliable knowledge in order to allow her to take a conscious decision on further management. From the moment of taking a decision to start the treatment and maintain pregnancy, the patient must be provided with the multidisciplinary 
care of highly qualified doctors cooperating with each other, who know and understand the complexity of coexistence of cancer and pregnancy [3, 4, 6, 9, 11-13].

Thanks to the publications in recent years, summarised in this paper, it is possible to avoid making hasty, tragic decisions enabling pregnant women affected by cancer to fight not only for their own health, but also for the birth of a healthy child.

\section{References}

1. Parazzini F. Cancer and pregnancy, the burden of the problem. Cancer and Pregnancy symposium in Milan - Abstract Book. Apr 2012

2. Kesic V. European survey on cancer and pregnancy: the ESGO data. Cancer and Pregnancy symposium in Milan - Abstract Book. Apr 2012.

3. Morice P, Uzan C, Gouy S, Verschraegen C, Haie-Meder C. Malignancies in pregnancy 1 . Gynaecological cancers in pregnancy. Lancet 2012; 379: 558-69.

4. Han SN, Verheecke M, Vandenbroucke T, Gziri MM, Van Calsteren K, Amant F. Management of gynecological cancers during pregnancy. Curr Oncol Rep 2014; 16: 415.

5. Ilancheran A, Low J, Ng JS. Gynaecological cancer in pregnancy. Best Pract Res Clin Obstet Gynaecol 2012; 26: 371-7.

6. Amant F, Halaska MJ, Fumagalli M, et al. Gynecologic cancers in pregnancy: guidelines of a second international consensus meeting. Int J Gynecol Cancer 2014; 24: 394-403.

7. Balleyguier C, Fournet C, Ben Hassen W, et al. Management of cervical cancer detected during pregnancy: role of magnetic resonance imaging. Clin Imaging 2012; 37: 70-6.

8. Hoellen F, Reibke R, Hornemann K, Thill M, Luedders DW, Kelling K, Hornemann A, Bohlmann MK. Cancer in pregnancy. Part I: basic diagnostic and therapeutic principles and treatment of gynaecological malignancies. Arch Gynecol Obstet 2012; 285: 195-205.

9. Amant F, Van Calsteren K, Vergote I, Ottevanger N. Gynaecologic oncology in pregnancy. Crit Rev Oncol Hematol 2008; 67: 187-95.

10. de Haan J, Verheecke M, Amant F. Management of ovarian cyst and cancer in pregnancy. Facts Views Vis Obgyn 2015; 7: 25-31.

11. Amant F, Van Calsteren K, Halaska MJ, et al. Gynecologic cancers in pregnancy: guidelines of an international consensus meeting. Int J Gynecol Cancer 2009; 19: S1-S12.

12. Surbone A, Peccatori F, Pavlidis N (eds.). Cancer and pregnancy. Springer-Verlag, Berlin Heidelberg 2008.

13. Morice P, Uzan C, Uzan S. Cancer In pregnancy: a challenging conflict of interest. Lancet 2012; 379: 495-6.

\section{Address for correspondence}

\section{Anna Skrzypczyk-Ostaszewicz}

Department of Oncology and Internal Medicine

Oncology Centre - The Maria Skłodowska-Curie Institute

Roentgena 5

02-781 Warsaw, Poland

phone: +48225462567

e-mail: anna.skrzypczyk@gmail.com

Submitted: 21.05 .2015

Accepted: $\quad 9.09 .2015$ 